\title{
Probing Phonon Polaritons Across Nanoscale Gaps
}

Isobel Bicket ${ }^{1}$, Connor Wong ${ }^{2}$, Joshua Tefal ${ }^{3}$, Nabil Bassim $^{4}$ and Maureen Joel Lagos ${ }^{5}$

${ }^{1}$ Canadian Centre for Electron Microscopy, McMaster University, Hamilton, Ontario, Canada, Hamilton, Ontario, Canada, ${ }^{2}$ Department of Engineering Physics, McMaster University, Hamilton, Ontario, Canada, United States, ${ }^{3}$ Department of Materials Science and Engineering, McMaster University, Hamilton, Ontario, Canada, United States, ${ }^{4}$ Department of Materials Science and Engineering, McMaster University, Hamilton, Ontario, Canada, Hamilton, Ontario, Canada, ${ }^{5}$ Department of Materials Science and Engineering, McMaster University, Hamilton, Canada, Canada

Over the last fifteen years, we have witnessed the rise of surface phonon polaritons as an alternative for enhancing light-matter interactions in nanoscale structures [1-3]. Due to this effect, infrared phononic cavities can be used for the implementation of platforms for nanoscale heat transfer and polaritonic chemistry [4-6]. These areas have offered new opportunities to investigate novel physical and chemical processes in extremely confined spaces. In spite of recent progress in these fields, there is still a lack of experimental studies regarding the phonon polariton behavior of a single nanoscale cavity. In this work, we use spatially-resolved vibrational EELS $[7,8]$ to locally study the properties of empty infrared cavities, seeking to understand the role of gap size and shape on the phonon polariton response and on the thermal properties of the cavity.

We fabricated infrared nanocavities (i.e. nanogaps) in suspended polycrystalline $\mathrm{SiC}$ membranes using a plasma FIB (Helios G4) equipped with a Xe+ plasma source. This fabrication also results in an amorphous $\sim 10 \mathrm{~nm}$ coating layer on the structures. The fabricated structures mainly consist of a suspended rod-like bridge $10 \mu \mathrm{m}$ long, which is cut in the middle to form a nanoscale gap (see for instance Fig. 1(b)). Fabricated gap sizes vary from $\sim 50$ to $280 \mathrm{~nm}$. The temperature of the membrane can be increased from room temperature to $\sim 1000^{\circ} \mathrm{C}$. Increasing the temperature causes the nanorod tips to move closer together, shrinking the gap size. We used a Nion UltraStem equipped with an aberration corrector and monochromator to study the phonon polaritonic response in nanogaps using a $\sim 1.5-2 \AA$ probe with an energy resolution of $\sim 10 \mathrm{meV}$, at $60 \mathrm{kV}$. Our EELS simulations were performed using the MNPBEM toolbox in Matlab $\mathbb{R}$ [9] with the $\mathrm{SiC}$ dielectric function from reference 5 .

The Reststrahlen band of a single $\mathrm{SiC}$ nanorod, extending from 98.8 to $120.4 \mathrm{meV}$, supports a wide variety of surface phonon polariton resonances, including Fabry-Perot (FP) resonances, transverse cap modes dependent on the shape of the rod tips, and high energy transverse cylinder modes dependent on the cross-sectional geometry. In a single SiC nanorod, we experimentally observe an EELS peak near $100 \mathrm{meV}$ under aloof excitation, with a full width at half maximum (FWHM) of $\sim 16.5 \mathrm{meV}$ (Fig. 1(a)). The small damping parameter of $\mathrm{SiC}$ in this energy region $(<0.5 \mathrm{meV})$ convolved with the FWHM of the energy distribution of the electron probe do not account for the width of the peak seen in the EELS data, nor for the asymmetry seen in the high energy tail of the peak. This might suggest that the electron beam is exciting higher order multipole resonances in addition to a dipolar mode similar to the well-known FP dipole mode of an isolated rod.

In a system with two rods forming a $280 \mathrm{~nm}$ nanogap, the experimental EELS peak is broader on the high energy side (FWHM $\sim 18 \mathrm{meV}$ ) compared to the single rod, and the apparent peak maximum blueshifts (Fig. 1(a)). Simulations on the EELS response of a model system consisting of two unsupported cylindrical nanorods confirm that the coupling between two nanorods in close proximity increases the EELS loss probability in the gap between the tips from high energy polaritonic multipole resonances (Fig. 2(a)). Reducing the gap distance causes energy splitting of bonding and antibonding FP modes, and coupling of cap modes and the higher 
energy transverse cylinder modes (Fig. 2(b)(i-iv)). At very small gap distances relative to the size of the nanorod, additional high energy peaks appear in the simulated spectrum (Fig. 2(b)(v)), exhibiting highly localized EELS signal directly in the nanogap. Experimental EELS spectra of an extremely small gap of $5 \mathrm{~nm}$ also show increased intensity on the higher energy side of the peak, between 110-115 meV (Fig. 1(b)), which may indicate the presence of this high energy localized gap mode.

The EELS scattering probability across the $280 \mathrm{~nm}$ gap (ADF shown in Fig. 1(a)) exhibits a parabolic distribution (Fig. 1(c)) at room temperature. Upon increasing the temperature of the membrane, the gap between the nanorod tips shrinks from $280 \mathrm{~nm}$ to $230 \mathrm{~nm}$ and a distinct tilt to the parabolic distribution is observed. Most of the points follow this trend nicely, save for one point halfway across the gap; the unexpectedly low value of this point may be due to software errors during the acquisition process. The asymmetrically designed nanorod pair shows a higher increase in scattering probability on one side of the gap relative to the other side at high temperatures. As the temperature increases, the thermal population of phonon modes increases, thereby increasing the EELS scattering probability; the intensity differences across this high temperature gap imply a difference in temperature on the two sides of the gap, opening up further avenues of study into heat transfer across this gap.

In summary, we are studying the coupling of surface phonon modes in nanorods across nanocavities of varying sizes and at different temperatures. Our work brings fundamental insights into the polaritonic response present in nanoscale cavities, representing progress in fields such as terahertz nanophotonics and nanoscale heat transport.

Acknowledgement: For financial support, M.J.L. and N.D.B acknowledges the Natural Sciences and Engineering Research Council (NSERC) of Canada under a Discovery Grant. CCEM instrumentation funding was provided by the Canada Foundation for Innovation. We thank Prof. P. Batson for access to the Nion UltraStem at Rutgers University, used to acquire the experimental EELS data. Corresponding author: mjlagos@mcmaster.ca.
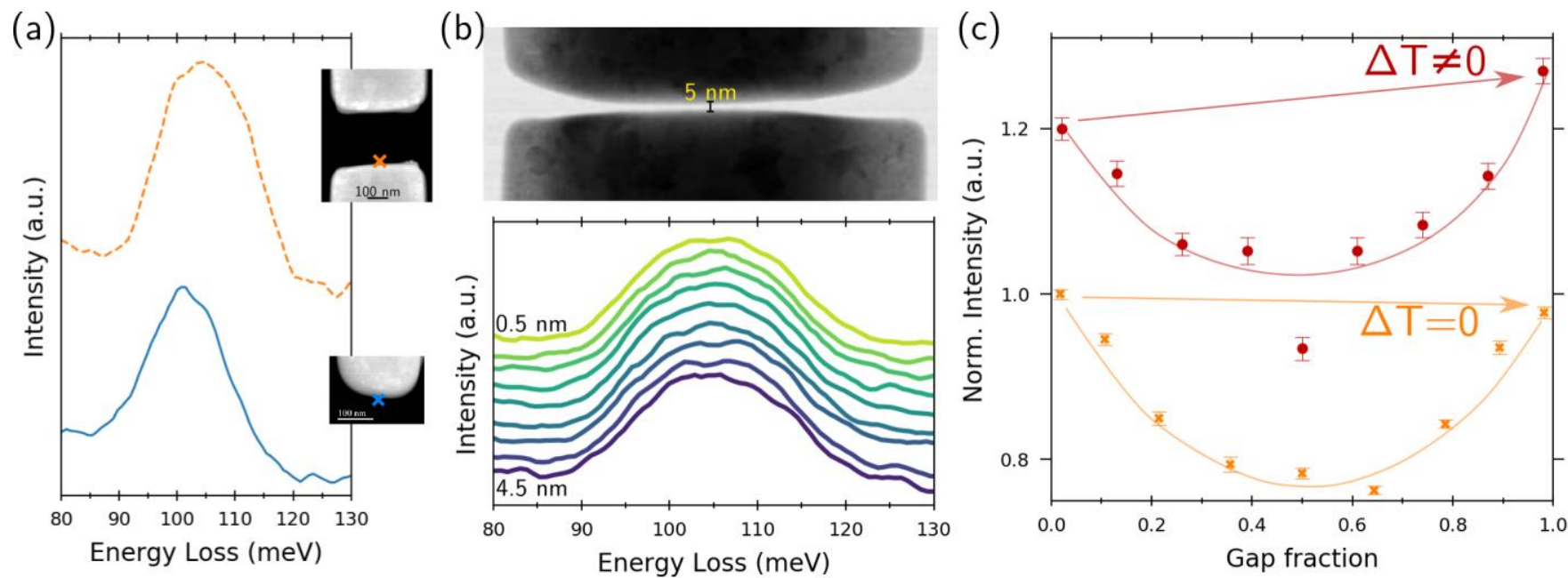

Figure 1. Experimental EELS results for SiC nanorods. (a) Spectra acquired with a $5 \mathrm{~nm}$ impact parameter (as indicated in the ADF inset images), offset for clarity, for a single $\mathrm{SiC}$ nanorod (blue) and a pair of $\mathrm{SiC}$ nanorods (orange) separated by a gap of $280 \mathrm{~nm}$ at $24^{\circ} \mathrm{C}$. (b) BF image (top) and line-scan spectra (bottom) across a 5 $\mathrm{nm}$ gap between a pair of $\mathrm{SiC}$ nanorods at $400^{\circ} \mathrm{C}$, with impact parameters from $0.5 \mathrm{~nm}$ (top of the gap) to 4.5 $\mathrm{nm}$ (bottom of the gap). (c) EELS intensity, averaged between 101-105 meV, scanned from the bottom to the 
top of the gap of the asymmetrically designed nanorod pair shown in (a), at room temperature (orange) and heated to $600^{\circ} \mathrm{C}$ (red), offset for clarity; parabolic lines and arrows are sketched as a guide to the eye.

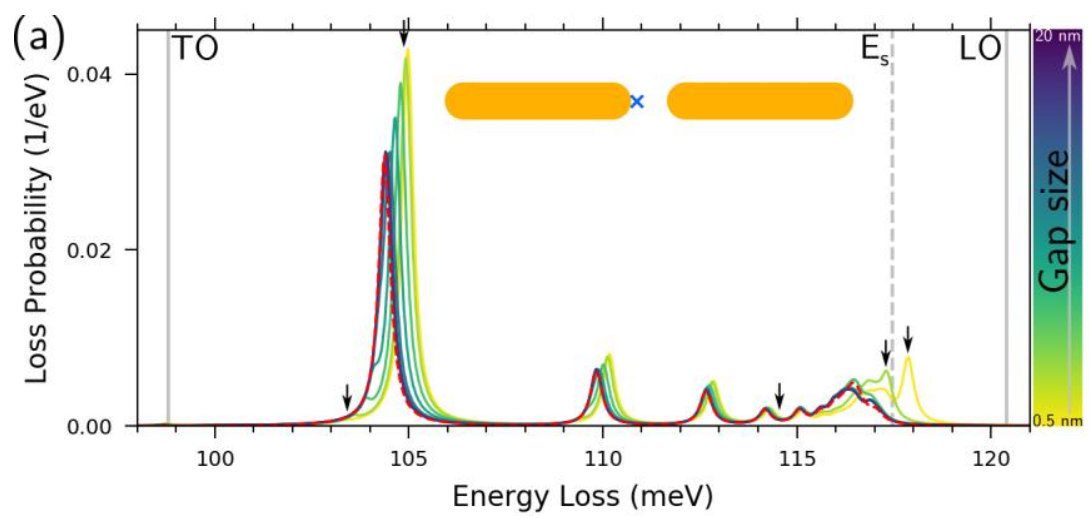

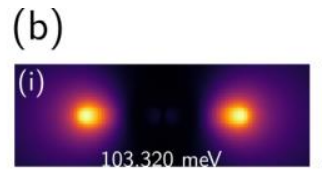
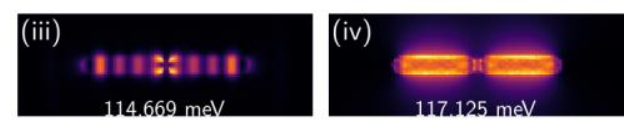

(v)

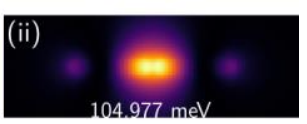

Figure 2. Calculated EELS intensity for a pair of $20 \mathrm{~nm}$ long round cylindrical nanowires with a diameter of $4 \mathrm{~nm}$. (a) Calculated spectra across the Reststrahlen band of $\mathrm{SiC}$ for decreasing gap size from $20 \mathrm{~nm}$ (blue) to $0.5 \mathrm{~nm}$ (yellow), with the electron beam $0.1 \mathrm{~nm}$ outside the tip of one nanowire; the red curve represents the response of a single cylindrical nanowire at a comparable impact parameter. (b) Calculated spatial EELS maps for a pair of nanorods with a $0.5 \mathrm{~nm}$ gap between the tips, showing the (i) bonding and (ii) antibonding FabryPerot modes; (iii) a coupled cap mode; (iv) a transverse cylinder mode; and (v) a highly localized mode in the gap between the tips.

\section{References}

[1] R. Hillenbrand et al., Nature, 418, 6894, 159-162, Jul. 2002.

2] M. S. Anderson, Appl. Phys. Lett., 83, 14, 2964-2966, Sep. 2003.

[3] M. Autore et al., Light: Science \& Applications, 7, 4, 17172, Apr. 2018.

[4] D.-Z. A. Chen et al., Phys. Rev. B, 72, 15, 155435, Oct. 2005.

[5] T. Wang et al., ACS Photonics, 4, 7, 1753-1760, Jul. 2017.

[6] L. Tranchant et al., Nano Lett., 19, 10, 6924-6930, Oct. 2019.

[7] M. J. Lagos et al., Nature, 543, 7646, pp. 529-532, Mar. 2017.

[8] M. J. Lagos and P. E. Batson, Nano Lett., 18, 7, 4556-4563, Jul. 2018.

[9] U. Hohenester, Comput. Phys. Commun., 185, 3, pp. 1177-1187, Mar. 2014. 